\title{
Some inequalities on the spectral radius of matrices
}

\author{
Linlin Zhao ${ }^{* *}$ and Qingbing Liu²
}

"Correspondence:

zhaolinlin0635@163.com

${ }^{1}$ College of Mathematics Science,

Dezhou University, Dezhou,

Shandong 253023, People's

Republic of China

Full list of author information is

available at the end of the article

\begin{abstract}
Let $A_{1}, A_{2}, \ldots, A_{k}$ be nonnegative matrices. In this paper, some upper bounds for the spectral radius $\rho\left(A_{1} \circ A_{2} \circ \cdots \circ A_{k}\right)$ are proposed. These bounds generalize some existing results, and comparisons between these bounds are also considered.
\end{abstract}

MSC: 15A06; 15A42; 15B34

Keywords: spectral radius; nonnegative matrix; Hadamard product

\section{Introduction}

Let $M_{n}$ denote the set of all $n \times n$ complex matrices and $A=\left(a_{i j}\right), B=\left(b_{i j}\right) \in M_{n}$. If $a_{i j}-b_{i j} \geq$ 0 , we say that $A \geq B$, and if $a_{i j} \geq 0$, we say that $A$ is nonnegative, denoted by $A \geq 0$. The symbol $\rho(A)$ stands for the spectral radius of $A$. If $A$ is a nonnegative matrix, the PerronFrobenius theorem guarantees that $\rho(A) \in \sigma(A)$, where $\sigma(A)$ denotes the spectrum of $A$.

If there does not exist a permutation matrix $P$ such that

$$
P^{T} A P=\left(\begin{array}{cc}
A_{1} & A_{12} \\
0 & A_{2}
\end{array}\right)
$$

where $A_{1}, A_{2}$ are square matrices, then $A$ is called irreducible.

Let $A$ be an irreducible nonnegative matrix. It is well known that there exists a positive vector $u$ such that $A u=\rho(A) u, u$ being called a right Perron eigenvector of $A$.

The Hadamard product of $A, B$ is defined as $A \circ B=\left(a_{i j} b_{i j}\right) \in M_{n}$.

Let $A \geq 0, B \geq 0$. By using the Gersgorin theorem, Brauer theorem and Brualdi theorem, respectively, the authors of [1-5] have given some inequalities for the upper bounds of $\rho(A \circ B)$. Audenaert [6], Horn and Zhang [7] proved a beautiful inequality on $\rho(A \circ B)$ for nonnegative matrices $A$ and $B$, that is, $\rho(A \circ B) \leq \rho(A B)$. Huang [8] generalized the above inequality to any $k$ nonnegative matrices, that is, $\rho\left(A_{1} \circ A_{2} \circ \cdots \circ A_{k}\right) \leq \rho\left(A_{1} A_{2} \cdots A_{k}\right)$. Motivated by [8] and [1-4, 9, 10], in this paper we propose some inequalities on the upper bounds for the spectral radius of the Hadamard product of any $k$ nonnegative matrices. These bounds generalize some existing results, and some comparisons between these bounds are also considered.

\section{Main results}

First, we give some lemmas which are useful for obtaining the main results.

(c) The Author(s) 2018. This article is distributed under the terms of the Creative Commons Attribution 4.0 International License (http://creativecommons.org/licenses/by/4.0/), which permits unrestricted use, distribution, and reproduction in any medium, provided you give appropriate credit to the original author(s) and the source, provide a link to the Creative Commons license, and indicate if changes were made. 
Lemma 2.1 ([11]) Let $A \in M_{n}$ be a nonnegative matrix. If $A_{k}$ is a principal submatrix of $A$, then $\rho\left(A_{k}\right) \leq \rho(A)$. If $A$ is irreducible and $A_{k} \neq A$, then $\rho\left(A_{k}\right)<\rho(A)$.

Lemma 2.2 ([11]) If $A \in M_{n}$ is an irreducible nonnegative matrix, and $A z \leq k z$ for a nonzero nonnegative vector $z$, then $\rho(A) \leq k$.

Lemma 2.3 ([12]) Let $A=\left(a_{i j}\right) \in M_{n}$ be a nonnegative matrix. Then

$$
\rho(A) \leq \max _{i \neq j} \frac{1}{2}\left\{a_{i i}+a_{j j}+\left[\left(a_{i i}-a_{j j}\right)^{2}+4 \sum_{k \neq i} a_{i k} \sum_{k \neq j} a_{j k}\right]^{\frac{1}{2}}\right\} .
$$

Lemma 2.4 Let $A_{1}, A_{2}, \ldots, A_{k} \in M_{n}$ and $D_{1}, D_{2}, \ldots, D_{k}$ be diagonal matrices of order $n$, then

$$
\begin{aligned}
& D^{-1}\left(A_{1} \circ A_{2} \circ \cdots \circ A_{k}\right) D \\
& \quad=\left(D_{1}^{-1} A_{1} D_{1}\right) \circ\left(D_{2}^{-1} A_{2} D_{2}\right) \circ \cdots \circ\left(D_{k}^{-1} A_{k} D_{k}\right),
\end{aligned}
$$

where $D$ equals the product of the matrices $D_{k}, D_{k-1}, \ldots, D_{1}$, that is, $D=D_{k} \cdots D_{2} D_{1}$.

Proof Let $d_{r, i}$ be the $i$ th diagonal of $D_{r}$ and $a_{r, i j}$ be the $(i, j)$ entry of $A_{r}(r=1,2, \ldots, k)$. Then the $(i, j)$ entry of $D^{-1}\left(A_{1} \circ A_{2} \circ \cdots \circ A_{k}\right) D$ is

$$
\frac{1}{\prod_{r=1}^{k} d_{r, i}}\left(\prod_{r=1}^{k} a_{r, i j}\right) \prod_{r=1}^{k} d_{r, j}=\prod_{r=1}^{k}\left(\frac{1}{d_{r, i}} a_{r, i j} d_{r, j}\right),
$$

which coincides with the $(i, j)$ entry of $\left(D_{1}^{-1} A_{1} D_{1}\right) \circ\left(D_{2}^{-1} A_{2} D_{2}\right) \circ \cdots \circ\left(D_{k}^{-1} A_{k} D_{k}\right)$. The proof is completed.

Theorem 2.1 Let $A_{1}, A_{2}, \ldots, A_{k} \in M_{n}$ and $A_{1}=\left(a_{i j}\right) \geq 0, A_{2}=\left(b_{i j}\right) \geq 0, \ldots, A_{k}=\left(k_{i j}\right) \geq 0$. Then

$$
\begin{aligned}
& \rho\left(A_{1} \circ A_{2} \circ \cdots \circ A_{k}\right) \\
& \quad \leq \max _{1 \leq i \leq n}\left\{a_{i i} b_{i i} \cdots k_{i i}+\left(\rho\left(A_{1}\right)-a_{i i}\right)\left(\rho\left(A_{2}\right)-b_{i i}\right) \cdots\left(\rho\left(A_{k}\right)-k_{i i}\right)\right\} .
\end{aligned}
$$

Proof If $A_{1} \circ A_{2} \circ \cdots \circ A_{k}$ is irreducible, then $A_{1}, A_{2}, \ldots, A_{k}$ are all irreducible. From Lemma 2.1, we have

$$
\rho\left(A_{1}\right)-a_{i i}>0, \quad \rho\left(A_{2}\right)-b_{i i}>0, \quad \ldots, \quad \rho\left(A_{k}\right)-k_{i i}>0, \quad \forall i \in N .
$$

Since $A_{1}, A_{2}, \ldots, A_{k}$ are nonnegative irreducible, there exist $k$ positive vectors $u, v, \ldots, w$ such that $A_{1} u=\rho\left(A_{1}\right) u, A_{2}^{T} v=\rho\left(A_{2}\right) v, \ldots, A_{k}^{T} w=\rho\left(A_{k}\right) w$. Thus, we have

$$
\begin{aligned}
& a_{i i} u_{i}+\sum_{j \neq i} a_{i j} u_{j}=\rho\left(A_{1}\right) u_{i}, \quad \forall i \in N, \\
& b_{i j} v_{j}+\sum_{i \neq j} b_{i j} v_{i}=\rho\left(A_{2}\right) v_{j}, \quad \forall j \in N,
\end{aligned}
$$




$$
k_{j j} w_{j}+\sum_{i \neq j} k_{i j} w_{i}=\rho\left(A_{k}\right) w_{j}, \quad \forall j \in N
$$

Thus, we have

$$
b_{i j} \leq \frac{\left[\rho\left(A_{2}\right)-b_{j j}\right] v_{j}}{v_{i}}, \quad \ldots, \quad k_{i j} \leq \frac{\left[\rho\left(A_{k}\right)-k_{j j}\right] w_{j}}{w_{i}} .
$$

Let $z$ be the vector $\left(z_{i}\right)$, where

$$
z_{i}=\frac{u_{i}}{\left(\rho\left(A_{2}\right)-b_{i i}\right) v_{i} \cdots\left(\rho\left(A_{k}\right)-k_{i i}\right) w_{i}}>0, \quad \forall i \in N
$$

We define $P=A_{1} \circ A_{2} \circ \cdots \circ A_{k}$. For any $i \in N$,

$$
\begin{aligned}
(P z)_{i} & =a_{i i} b_{i i} \cdots k_{i i} z_{i}+\sum_{i \neq j} a_{i j} b_{i j} \cdots k_{i j} z_{j} \\
& \leq a_{i i} b_{i i} \cdots k_{i i} z_{i}+\sum_{i \neq j} a_{i j} \frac{\left(\rho\left(A_{2}\right)-b_{j j}\right) v_{j}}{v_{i}} \cdots \frac{\left(\rho\left(A_{k}\right)-k_{j j}\right) w_{j}}{w_{i}} z_{j} .
\end{aligned}
$$

For

$$
z_{j}=\frac{u_{j}}{\left(\rho\left(A_{2}\right)-b_{j j}\right) v_{j} \cdots\left(\rho\left(A_{k}\right)-k_{j j}\right) w_{j}}
$$

we have

$$
\begin{aligned}
(P z)_{i} & \leq a_{i i} b_{i i} \cdots k_{i i} z_{i}+\frac{1}{v_{i} \cdots w_{i}} \sum_{i \neq j} a_{i j} u_{j} \\
& =a_{i i} b_{i i} \cdots k_{i i} z_{i}+\frac{1}{v_{i} \cdots w_{i}}\left(\rho\left(A_{1}\right)-a_{i i}\right) u_{i} \\
& =a_{i i} b_{i i} \cdots k_{i i} z_{i}+\left(\rho\left(A_{2}\right)-b_{i i}\right) \cdots\left(\rho\left(A_{k}\right)-k_{i i}\right)\left(\rho\left(A_{1}\right)-a_{i i}\right) z_{i} \\
& =\left\{a_{i i} b_{i i} \cdots k_{i i}+\left(\rho\left(A_{1}\right)-a_{i i}\right)\left(\rho\left(A_{2}\right)-b_{i i}\right) \cdots\left(\rho\left(A_{k}\right)-k_{i i}\right)\right\} z_{i} .
\end{aligned}
$$

By Lemma 2.2, this shows that

$$
\rho\left(A_{1} \circ \cdots \circ A_{k}\right) \leq \max _{1 \leq i \leq n}\left\{a_{i i} b_{i i} \cdots k_{i i}+\left(\rho\left(A_{1}\right)-a_{i i}\right)\left(\rho\left(A_{2}\right)-b_{i i}\right) \cdots\left(\rho\left(A_{k}\right)-k_{i i}\right)\right\} .
$$

If $A_{1} \circ A_{2} \circ \cdots \circ A_{k}$ is reducible, we denote by $P=\left(p_{i j}\right)$ the $n \times n$ permutation matrix with $p_{12}=p_{23}=\cdots=p_{n 1}=1$, the remaining $p_{i j}=0$, then all $A_{1}+t P, A_{2}+t P, \ldots, A_{k}+t P$ are nonnegative irreducible matrices for any chosen positive real numbers $t$. We substitute $A_{1}+t P, A_{2}+t P, \ldots, A_{k}+t P$ for $A_{1}, A_{2}, \ldots, A_{k}$, respectively, in the previous case, and then, letting $t \rightarrow 0$, the result follows by continuity. The proof is completed.

Setting $k=2$ in Theorem 2.1, we have the following corollary. 
Corollary 2.1 ([1]) Let $A_{1}, A_{2} \in M_{n}$ and $A_{1} \geq 0, A_{2} \geq 0$. Then

$$
\rho\left(A_{1} \circ A_{2}\right) \leq \max _{1 \leq i \leq n}\left\{a_{i i} b_{i i}+\left(\rho\left(A_{1}\right)-a_{i i}\right)\left(\rho\left(A_{2}\right)-b_{i i}\right)\right\} .
$$

Theorem 2.2 Let $A_{1}, A_{2}, \ldots, A_{k} \in M_{n}$ and $A_{1}=\left(a_{i j}\right) \geq 0, A_{2}=\left(b_{i j}\right) \geq 0, \ldots, A_{k}=\left(k_{i j}\right) \geq 0$.

Then

$$
\begin{aligned}
& \rho\left(A_{1} \circ A_{2} \circ \cdots \circ A_{k}\right) \\
& \leq \max _{i \neq j} \frac{1}{2}\left\{a_{i i} b_{i i} \cdots k_{i i}+a_{i j} b_{i j} \cdots k_{j j}+\left[\left(a_{i i} b_{i i} \cdots k_{i i}-a_{j j} b_{j j} \cdots k_{j j}\right)^{2}\right.\right. \\
& \left.\left.\quad+4\left(\rho\left(A_{1}\right)-a_{i i}\right) \cdots\left(\rho\left(A_{k}\right)-k_{i i}\right)\left(\rho\left(A_{1}\right)-a_{j j}\right) \cdots\left(\rho\left(A_{k}\right)-k_{j j}\right)\right]^{\frac{1}{2}}\right\} .
\end{aligned}
$$

Proof First we assume that $A_{1} \circ A_{2} \circ \cdots \circ A_{k}$ is irreducible. Obviously, $A_{1}, A_{2}, \ldots, A_{k}$ are all irreducible, from Lemma 2.1, we have

$$
\rho\left(A_{1}\right)-a_{i i}>0, \quad \rho\left(A_{2}\right)-b_{i i}>0, \quad \ldots, \quad \rho\left(A_{k}\right)-k_{i i}>0, \quad \forall i \in N .
$$

For the irreducibility of $A_{1}, A_{2}, \ldots, A_{k}$, there exist $k$ positive vectors $u=\left(u_{i}\right), v=$ $\left(v_{i}\right), \ldots, w=\left(w_{i}\right)$ such that $A_{1} u=\rho\left(A_{1}\right) u, A_{2} v=\rho\left(A_{2}\right) v, \ldots, A_{k} w=\rho\left(A_{k}\right) w$. Thus, we have

$$
\begin{aligned}
& a_{i i}+\sum_{j \neq i} \frac{a_{i j} u_{j}}{u_{i}}=\rho\left(A_{1}\right), \quad \forall i \in N, \\
& b_{i i}+\sum_{j \neq i} \frac{b_{i j} v_{j}}{v_{i}}=\rho\left(A_{2}\right), \quad \forall i \in N, \\
& \ldots, \quad \\
& k_{i i}+\sum_{j \neq i} \frac{k_{i j} w_{j}}{w_{i}}=\rho\left(A_{k}\right), \quad \forall i \in N .
\end{aligned}
$$

Define

$$
\begin{aligned}
& U=\operatorname{diag}\left(u_{1}, u_{2}, \ldots, u_{n}\right), \quad V=\operatorname{diag}\left(v_{1}, v_{2}, \ldots, v_{n}\right), \\
& W=\operatorname{diag}\left(w_{1}, w_{2}, \ldots, w_{n}\right) .
\end{aligned}
$$

Let

$$
\begin{aligned}
& \hat{A}_{1}=\left(\hat{a}_{i j}\right)=U^{-1} A_{1} U=\left(\frac{1}{u_{i}} a_{i j} u_{j}\right), \\
& \hat{A}_{2}=\left(\hat{b}_{i j}\right)=V^{-1} A_{2} V\left(\frac{1}{v_{i}} b_{i j} v_{j}\right), \\
& \ldots, \\
& \hat{A}_{k}=\left(\hat{k}_{i j}\right)=W^{-1} A_{k} W\left(\frac{1}{w_{i}} k_{i j} w_{j}\right) .
\end{aligned}
$$


It is easy to show that $\hat{A}_{1}, \hat{A}_{2}, \ldots, \hat{A}_{k}$ are all nonnegative irreducible matrices, and all the row sums of $\hat{A}_{1}, \hat{A}_{2}, \ldots, \hat{A}_{k}$ are equal to $\rho\left(A_{1}\right), \rho\left(A_{2}\right), \ldots, \rho\left(A_{k}\right)$, respectively.

Let $D=W \cdots V U$ be the product of $k$ nonsingular diagonal matrices $U, V, \ldots, W$. According to Lemma 2.4, we have

$$
\begin{aligned}
& D^{-1}\left(A_{1} \circ A_{2} \circ \cdots \circ A_{k}\right) D \\
& \quad=\left(U^{-1} A_{1} U\right) \circ\left(V^{-1} A_{2} V\right) \circ \cdots \circ\left(W^{-1} A_{k} W\right) \\
& \quad=\hat{A}_{1} \circ \hat{A}_{2} \circ \cdots \circ \hat{A}_{k} .
\end{aligned}
$$

Thus, we have $\rho\left(A_{1} \circ A_{2} \circ \cdots \circ A_{k}\right)=\rho\left(\hat{A}_{1} \circ \hat{A}_{2} \circ \cdots \circ \hat{A}_{k}\right)$. From Lemma 2.3, we have

$$
\begin{aligned}
\rho\left(\hat{A}_{1} \circ \hat{A}_{2} \circ \cdots \circ \hat{A}_{k}\right) \\
\leq \max _{i \neq j} \frac{1}{2}\left\{\hat{a}_{i i} \hat{b}_{i i} \cdots \hat{k}_{i i}+\hat{a}_{j j} \hat{b}_{j j} \cdots \hat{k}_{j j}+\left[\left(\hat{a}_{i i} \hat{b}_{i i} \cdots \hat{k}_{i i}-\hat{a}_{j j} \hat{b}_{j j} \cdots \hat{k}_{j j}\right)^{2}\right.\right. \\
\left.\left.\quad+4\left(\sum_{k \neq i} \hat{a}_{i k} \hat{b}_{i k} \cdots \hat{k}_{i k}\right)\left(\sum_{k \neq j} \hat{a}_{j k} \hat{b}_{j k} \cdots \hat{k}_{j k}\right)\right]^{\frac{1}{2}}\right\} \\
\leq \max _{i \neq j} \frac{1}{2}\left\{a_{i i} b_{i i} \cdots k_{i i}+a_{j j} b_{j j} \cdots k_{j j}+\left[\left(a_{i i} b_{i i} \cdots k_{i i}-a_{j j} b_{i j} \cdots k_{j j}\right)^{2}\right.\right. \\
\left.\left.\quad+4\left(\sum_{k \neq i} \frac{a_{i k} u_{k}}{u_{i}} \sum_{k \neq i} \frac{b_{i k} v_{k}}{v_{i}} \cdots \sum_{k \neq i} \frac{k_{i k} w_{k}}{w_{i}}\right)\left(\sum_{k \neq j} \frac{a_{j k} u_{k}}{u_{j}} \sum_{k \neq j} \frac{b_{i k} v_{k}}{v_{j}} \cdots \sum_{k \neq j} \frac{k_{j k} w_{k}}{w_{j}}\right)\right]^{\frac{1}{2}}\right\} \\
=\max _{i \neq j} \frac{1}{2}\left\{a_{i i} b_{i i} \cdots k_{i i}+a_{j j} b_{j j} \cdots k_{j j}+\left[\left(a_{i i} b_{i i} \cdots k_{i i}-a_{i j} b_{i j} \cdots k_{j j}\right)^{2}\right.\right. \\
\quad+4\left(\rho\left(A_{1}\right)-a_{i i}\right)\left(\rho\left(A_{2}\right)-b_{i i}\right) \cdots\left(\rho\left(A_{k}\right)-k_{i i}\right)\left(\rho\left(A_{1}\right)-a_{j j}\right) \\
\left.\left.\quad \times\left(\rho\left(A_{2}\right)-b_{j j}\right) \cdots\left(\rho\left(A_{k}\right)-k_{j j}\right)\right]^{\frac{1}{2}}\right\} .
\end{aligned}
$$

If $A_{1} \circ A_{2} \circ \cdots \circ A_{k}$ is reducible, the proof is similar to Theorem 2.1. So, the proof is completed.

Setting $k=2$ in Theorem 2.2, we have the following corollary.

Corollary 2.2 ([2]) Let $A_{1}, A_{2} \in M_{n}$ and $A_{1} \geq 0, A_{2} \geq 0$. Then

$$
\begin{aligned}
\rho\left(A_{1} \circ A_{2}\right) \leq & \max _{i \neq j} \frac{1}{2}\left\{a_{i i} b_{i i}+a_{j j} b_{i j}+\left[\left(a_{i i} b_{i i}-a_{i j} b_{j j}\right)^{2}\right.\right. \\
& \left.\left.+4\left(\rho\left(A_{1}\right)-a_{i i}\right)\left(\rho\left(A_{2}\right)-b_{i i}\right)\left(\rho\left(A_{1}\right)-a_{j j}\right)\left(\rho\left(A_{2}\right)-b_{j j}\right)\right]^{\frac{1}{2}}\right\} .
\end{aligned}
$$

We next give a simple comparison between the upper bound in (2.1) and the upper bound in (2.2). Without loss of generality, for $i \neq j$, assume that

$$
\begin{aligned}
& a_{i i} b_{i i} \cdots k_{i i}+\left(\rho\left(A_{1}\right)-a_{i i}\right)\left(\rho\left(A_{2}\right)-b_{i i}\right) \cdots\left(\rho\left(A_{k}\right)-k_{i i}\right) \\
& \geq a_{j j} b_{j j} \cdots k_{j j}+\left(\rho\left(A_{1}\right)-a_{j j}\right)\left(\rho\left(A_{2}\right)-b_{j j}\right) \cdots\left(\rho\left(A_{k}\right)-k_{j j}\right) .
\end{aligned}
$$


Let $\gamma=a_{i i} b_{i i} \cdots k_{i i}+a_{j j} b_{j j} \cdots k_{j j}$. From (2.2), we have

$$
\begin{aligned}
a_{i i} b_{i i} & \cdots k_{i i}+a_{j j} b_{j j} \cdots k_{j j}+\left[\left(a_{i i} b_{i i} \cdots k_{i i}-a_{j j} b_{j j} \cdots k_{j j}\right)^{2}\right. \\
& +4\left(\rho\left(A_{1}\right)-a_{i i}\right)\left(\rho\left(A_{2}\right)-b_{i i}\right) \cdots\left(\rho\left(A_{k}\right)-k_{i i}\right)\left(\rho\left(A_{1}\right)-a_{j j}\right) \\
& \left.\times\left(\rho\left(A_{2}\right)-b_{j j}\right) \cdots\left(\rho\left(A_{k}\right)-k_{j j}\right)\right]^{\frac{1}{2}} \\
\leq & \gamma+\left\{\left(a_{i i} b_{i i} \cdots k_{i i}-a_{j j} b_{j j} \cdots k_{j j}\right)^{2}+4\left(\rho\left(A_{1}\right)-a_{i i}\right) \cdots\left(\rho\left(A_{k}\right)-k_{i i}\right)\right. \\
& \left.\times\left[\left(\rho\left(A_{1}\right)-a_{i i}\right)\left(\rho\left(A_{2}\right)-b_{i i}\right) \cdots\left(\rho\left(A_{k}\right)-k_{i i}\right)+a_{i i} b_{i i} \cdots k_{i i}-a_{j j} b_{j j} \cdots k_{j j}\right]\right\}^{\frac{1}{2}} \\
=\gamma & +\left[\left(a_{i i} b_{i i} \cdots k_{i i}-a_{j j} b_{j j} \cdots k_{j j}+2\left(\rho\left(A_{1}\right)-a_{i i}\right) \cdots\left(\rho\left(A_{k}\right)-k_{i i}\right)\right)^{2}\right]^{\frac{1}{2}} \\
= & 2 a_{i i} b_{i i} \cdots k_{i i}+2\left(\rho\left(A_{1}\right)-a_{i i}\right)\left(\rho\left(A_{2}\right)-b_{i i}\right) \cdots\left(\rho\left(A_{k}\right)-k_{i i}\right) .
\end{aligned}
$$

Thus, we have

$$
\begin{aligned}
\rho( & \left.A_{1} \circ A_{2} \circ \cdots \circ A_{k}\right) \\
\leq & \max _{i \neq j} \frac{1}{2}\left\{a_{i i} \cdots k_{i i}+a_{j j} \cdots k_{j j}+\left[\left(a_{i i} \cdots k_{i i}-a_{j j} \cdots k_{j j}\right)^{2}+4\left(\rho\left(A_{1}\right)-a_{i i}\right)\right.\right. \\
& \left.\left.\times\left(\rho\left(A_{2}\right)-b_{i i}\right) \cdots\left(\rho\left(A_{k}\right)-k_{i i}\right)\left(\rho\left(A_{1}\right)-a_{j j}\right)\left(\rho\left(A_{2}\right)-b_{j j}\right) \cdots\left(\rho\left(A_{k}\right)-k_{j j}\right)\right]^{\frac{1}{2}}\right\} \\
\leq & \max _{1 \leq i \leq n} \frac{1}{2}\left[2 a_{i i} b_{i i} \cdots k_{i i}+2\left(\rho\left(A_{1}\right)-a_{i i}\right) \cdots\left(\rho\left(A_{k}\right)-k_{i i}\right)\right] \\
= & \max _{1 \leq i \leq n}\left[a_{i i} b_{i i} \cdots k_{i i}+\left(\rho\left(A_{1}\right)-a_{i i}\right)\left(\rho\left(A_{2}\right)-b_{i i}\right) \cdots\left(\rho\left(A_{k}\right)-k_{i i}\right)\right] .
\end{aligned}
$$

Hence, bound (2.2) is better than bound (2.1).

In [8], the author proved that

$$
\rho\left(A_{1} \circ A_{2} \circ \cdots \circ A_{k}\right) \leq \rho\left(A_{1} A_{2} \cdots A_{k}\right) .
$$

At present, we cannot give the comparison between bounds (2.1) and (2.3) or bounds (2.2) and (2.3), but the following numerical example shows that bounds (2.1) and (2.2) are better than (2.3). Next,we give an example: Consider four $4 \times 4$ nonnegative matrices

$$
\begin{array}{rlrl}
A & =\left(\begin{array}{cccc}
4 & 1 & 0 & 2 \\
0 & 0.05 & 1 & 1 \\
0 & 0 & 4 & 0.5 \\
1 & 0.5 & 0 & 4
\end{array}\right), & B=\left(\begin{array}{llll}
1 & 1 & 1 & 1 \\
1 & 1 & 1 & 1 \\
1 & 1 & 1 & 1 \\
1 & 1 & 1 & 1
\end{array}\right), \\
C & =\left(\begin{array}{cccc}
2 & 0 & 1 & 1 \\
1 & 4 & 0.5 & 0.5 \\
1 & 0 & 3 & 0.5 \\
0.5 & 1 & 1 & 2
\end{array}\right), & D & =\left(\begin{array}{cccc}
2 & 0.5 & 0.5 & 0.5 \\
1 & 1 & 1 & 1 \\
0.5 & 0 & 2 & 0.5 \\
0 & 1 & 1 & 2
\end{array}\right) .
\end{array}
$$

(i) It is easy to calculate that $\rho(A \circ B)=5.4983$. By inequalities (2.1) and (2.2), we have

$$
\rho(A \circ B) \leq \max _{1 \leq i \leq 4}\left\{a_{i i} b_{i i}+\left(\rho(A)-a_{i i}\right)\left(\rho(B)-b_{i i}\right)\right\}=16.3949
$$


and

$$
\rho(A \circ B) \leq 11.6478 \text {. }
$$

By inequality (2.3), we have

$$
\rho(A \circ B) \leq \rho(A B)=19.05 .
$$

(ii) From calculation, we get $\rho(A \circ B \circ C)=12.0014$. By inequalities (2.1) and (2.2), we have

$$
\rho(A \circ B \circ C) \leq \max _{1 \leq i \leq 4}\left\{a_{i i} b_{i i} c_{i i}+\left(\rho(A)-a_{i i}\right)\left(\rho(B)-b_{i i}\right)\left(\rho(C)-c_{i i}\right)\right\}=20.8846,
$$

and

$$
\rho(A \circ B \circ C) \leq 17.8268 .
$$

By inequality (2.3), we have

$$
\rho(A \circ B \circ C) \leq \rho(A B C)=88.5 \text {. }
$$

(iii) Let $A \circ B \circ C \circ D=G=\left(g_{i j}\right)$. Then

$$
G=\left(\begin{array}{cccc}
16 & 0 & 0 & 1 \\
0 & 0.2 & 0.5 & 0.5 \\
0 & 0 & 24 & 0.075 \\
0 & 0.5 & 0 & 16
\end{array}\right), \quad A B C D=\left(\begin{array}{cccc}
117.25 & 78.75 & 155.75 & 126 \\
34.3375 & 23.0625 & 45.6125 & 36.9 \\
75.375 & 50.625 & 100.125 & 81 \\
92.125 & 61.875 & 122.375 & 99
\end{array}\right)
$$

It is easy to calculate that $\rho(G)=24.0001$. By inequalities (2.1) and (2.2), we have

$$
\rho(G) \leq \max _{1 \leq i \leq 4}\left\{a_{i i} b_{i i} c_{i i} d_{i i}+\left(\rho(A)-a_{i i}\right)\left(\rho(B)-b_{i i}\right)\left(\rho(C)-c_{i i}\right)\left(\rho(D)-d_{i i}\right)\right\}=36.6608
$$

and

$$
\begin{aligned}
\rho(G) \leq & \max _{i \neq j} \frac{1}{2}\left\{g_{i i}+g_{i j}+\left[\left(g_{i i}-g_{j j}\right)^{2}+4\left(\rho(A)-a_{i i}\right)\left(\rho(B)-b_{i i}\right)\left(\rho(C)-c_{i i}\right)\right.\right. \\
& \left.\left.\times\left(\rho(D)-d_{i i}\right)\left(\rho(A)-a_{j j}\right)\left(\rho(B)-b_{j j}\right)\left(\rho(C)-c_{j j}\right)\left(\rho(D)-d_{j j}\right)\right]^{\frac{1}{2}}\right\} \\
= & 32.4451 .
\end{aligned}
$$

By inequality (2.3), we have $\rho(G) \leq \rho(A B C D)=339.44$.

Next, we will give some other inequalities for $\rho\left(A_{1} \circ A_{2} \circ \cdots \circ A_{k}\right)$. For $A_{1} \geq 0$, write $L_{1}=A_{1}-\operatorname{diag}\left(a_{11}, \ldots, a_{n n}\right)$. We denote $J_{A_{1}}=D_{1}^{-1} L_{1}$ with $D_{1}=\operatorname{diag}\left(d_{i i}\right)$, where

$$
d_{i i}= \begin{cases}a_{i i}, & \text { if } a_{i i} \neq 0 \\ 1, & \text { if } a_{i i}=0\end{cases}
$$

Then $J_{A_{1}}$ is nonnegative. 
For $A_{2} \geq 0$, let $D_{2}=\operatorname{diag}\left(s_{i i}\right), \ldots$, for $A_{k} \geq 0$, let $D_{k}=\operatorname{diag}\left(t_{i i}\right)$ with

$$
\begin{aligned}
& s_{i i}= \begin{cases}b_{i i}, & \text { if } b_{i i} \neq 0, \\
1, & \text { if } b_{i i}=0,\end{cases} \\
& \ldots, \\
& t_{i i}= \begin{cases}k_{i i}, & \text { if } k_{i i} \neq 0, \\
1, & \text { if } k_{i i}=0,\end{cases}
\end{aligned}
$$

respectively. Then the nonnegative matrix $J_{A_{2}}, \ldots, J_{A_{k}}$ can be similarly defined.

Theorem 2.3 Let $A_{1}, A_{2}, \ldots, A_{k} \in M_{n}$ and $A_{1} \geq 0, A_{2} \geq 0, \ldots, A_{k} \geq 0$. Then

$$
\begin{aligned}
& \rho\left(A_{1} \circ A_{2} \circ \cdots \circ A_{k}\right) \\
& \quad \leq \max _{1 \leq i \leq n}\left\{a_{i i} b_{i i} \cdots k_{i i}+d_{i i} \rho\left(J_{A_{1}}\right) s_{i i} \rho\left(J_{A_{2}}\right) \cdots t_{i i} \rho\left(J_{A_{k}}\right)\right\} .
\end{aligned}
$$

Proof Let $Q=A_{1} \circ A_{2} \circ \cdots \circ A_{k}$. First assume that $Q$ is irreducible. Obviously $A_{1}, A_{2}, \ldots, A_{k}$ are all irreducible, and then $J_{A_{1}}, J_{A_{2}}, \ldots, J_{A_{k}}$ are all nonnegative irreducible, so there exist $k$ positive vectors $x, y, \ldots, h$ such that $J_{A_{1}} x=\rho\left(J_{A_{1}}\right) x, J_{A_{2}} y=\rho\left(J_{A_{2}}\right) y, \ldots, J_{A_{k}} h=\rho\left(J_{A_{k}}\right) h$. So, we have

$$
\sum_{j \neq i} \frac{a_{i j} x_{j}}{x_{i}}=d_{i i} \rho\left(J_{A_{1}}\right), \quad \sum_{j \neq i} \frac{b_{i j} y_{j}}{y_{i}}=s_{i i} \rho\left(J_{A_{2}}\right), \quad \ldots, \quad \sum_{j \neq i} \frac{k_{i j} h_{j}}{h_{i}}=t_{i i} \rho\left(J_{A_{k}}\right) .
$$

Now let $z=\left(z_{i}\right)$ be the vector, where $z_{i}=\left(x_{i} y_{i} \cdots h_{i}\right)>0$ for all $i$. For the irreducible nonnegative matrix $Q$, we have

$$
\begin{aligned}
(Q z)_{i} & =a_{i i} b_{i i} \cdots k_{i i} z_{i}+\sum_{i \neq j} a_{i j} b_{i j} \cdots k_{i j} z_{j} \\
& \leq a_{i i} b_{i i} \cdots k_{i i} z_{i}+\left(\sum_{i \neq j} a_{i j} x_{j}\right)\left(\sum_{i \neq j} b_{i j} y_{j}\right) \cdots\left(\sum_{i \neq j} k_{i j} h_{j}\right) \\
& =a_{i i} b_{i i} \cdots k_{i i} z_{i}+\left(d_{i i} x_{i} \rho\left(J_{A_{1}}\right)\right)\left(s_{i i} y_{i} \rho\left(J_{A_{2}}\right)\right) \cdots\left(t_{i i} h_{i} \rho\left(J_{A_{k}}\right)\right) \\
& =\left\{a_{i i} b_{i i} \cdots k_{i i}+d_{i i} \rho\left(J_{A_{1}}\right) s_{i i} \rho\left(J_{A_{2}}\right) \cdots t_{i i} \rho\left(J_{A_{k}}\right)\right\} z_{i} .
\end{aligned}
$$

By Lemma 2.2, this shows that

$$
\rho\left(A_{1} \circ A_{2} \circ \cdots \circ A_{k}\right) \leq \max _{1 \leq i \leq n}\left\{a_{i i} b_{i i} \cdots k_{i i}+d_{i i} \rho\left(J_{A_{1}}\right) s_{i i} \rho\left(J_{A_{2}}\right) \cdots t_{i i} \rho\left(J_{A_{k}}\right)\right\} .
$$

The proof is completed.

Setting $k=2$ in Theorem 2.3, we have the following corollary.

Corollary 2.3 ([4]) Let $A_{1}, A_{2} \in M_{n}$ and $A_{1} \geq 0, A_{2} \geq 0$. Then

$$
\rho\left(A_{1} \circ A_{2}\right) \leq \max _{1 \leq i \leq n}\left\{a_{i i} b_{i i}+d_{i i} \rho\left(J_{A_{1}}\right) s_{i i} \rho\left(J_{A_{2}}\right)\right\}
$$


Theorem 2.4 Let $A_{1}, A_{2}, \ldots, A_{k} \in M_{n}$ and $A_{1} \geq 0, A_{2} \geq 0, \ldots, A_{k} \geq 0$. Then

$$
\begin{aligned}
& \rho\left(A_{1} \circ A_{2} \circ \cdots \circ A_{k}\right) \\
& \leq \max _{i \neq j} \frac{1}{2}\left\{a_{i i} b_{i i} \cdots k_{i i}+a_{j j} b_{j j} \cdots k_{j j}+\left[\left(a_{i i} b_{i i} \cdots k_{i i}-a_{j j} b_{j j} \cdots k_{j j}\right)^{2}\right.\right. \\
& \left.\left.\quad+4\left(d_{i i} s_{i i} \cdots t_{i i}\right)\left(d_{j j} s_{j j} \cdots t_{j j}\right)\left(\rho^{2}\left(J_{A_{1}}\right) \rho^{2}\left(J_{A_{2}}\right) \cdots \rho^{2}\left(J_{A_{k}}\right)\right)\right]^{\frac{1}{2}}\right\} .
\end{aligned}
$$

Proof First we assume that $A_{1} \circ A_{2} \circ \cdots \circ A_{k}$ is irreducible. Obviously, $J_{A_{1}}, J_{A_{2}}, \ldots, J_{A_{k}}$ are all nonnegative irreducible, then there exist $k$ positive vectors $x, y, \ldots, h$ such that $J_{A_{1}} x=$ $\rho\left(J_{A_{1}}\right) x, J_{A_{2}} y=\rho\left(J_{A_{2}}\right) y, \ldots, J_{A_{k}} h=\rho\left(J_{A_{k}}\right) h$. Thus, we have

$$
\sum_{j \neq i} \frac{a_{i j} x_{j}}{x_{i}}=d_{i i} \rho\left(J_{A_{1}}\right), \quad \sum_{j \neq i} \frac{b_{i j} y_{j}}{y_{i}}=s_{i i} \rho\left(J_{A_{2}}\right), \quad \ldots, \quad \sum_{j \neq i} \frac{k_{i j} h_{j}}{h_{i}}=t_{i i} \rho\left(J_{A_{k}}\right) .
$$

Define

$$
\begin{aligned}
& X=\operatorname{diag}\left(x_{1}, x_{2}, \ldots, x_{n}\right), \quad Y=\operatorname{diag}\left(y_{1}, y_{2}, \ldots, y_{n}\right), \quad \ldots, \\
& H=\operatorname{diag}\left(h_{1}, h_{2}, \ldots, h_{n}\right) .
\end{aligned}
$$

Let

$$
\tilde{A_{1}}=\left(\tilde{a}_{i j}\right)=X^{-1} A_{1} X, \quad \tilde{A}_{2}=\left(\tilde{b}_{i j}\right)=Y^{-1} A_{2} Y, \quad \ldots, \quad \tilde{A}_{k}=\left(\tilde{k}_{i j}\right)=H^{-1} A_{k} H
$$

From Lemma 2.4, we have

$$
\begin{aligned}
& \left(X^{-1} Y^{-1} \cdots H^{-1}\right)\left(A_{1} \circ A_{2} \circ \cdots \circ A_{k}\right)(H \cdots Y X) \\
& \quad=\left(X^{-1} A_{1} X\right) \circ\left(Y^{-1} A_{2} Y\right) \circ \cdots \circ\left(H^{-1} A_{k} H\right) \\
& \quad=\tilde{A_{1}} \circ \tilde{A_{2}} \circ \cdots \circ \tilde{A_{k}} .
\end{aligned}
$$

Thus, $\rho\left(A_{1} \circ A_{2} \circ \cdots \circ A_{k}\right)=\rho\left(\tilde{A_{1}} \circ \tilde{A_{2}} \circ \cdots \circ \tilde{A_{k}}\right)$. From Lemma 2.3, we have

$$
\begin{aligned}
\rho\left(\tilde{A_{1}} \circ \tilde{A_{2}} \circ \cdots \circ \tilde{A_{k}}\right) \\
\leq \max _{i \neq j} \frac{1}{2}\left\{a_{i i} b_{i i} \cdots k_{i i}+a_{j j} b_{j j} \cdots k_{j j}+\left[\left(a_{i i} b_{i i} \cdots k_{i i}-a_{j j} b_{j j} \cdots k_{j j}\right)^{2}\right.\right. \\
\left.\left.\quad+4\left(\sum_{k \neq i} \frac{a_{i k} x_{k}}{x_{i}} \frac{b_{i k} y_{k}}{y_{i}} \cdots \frac{k_{i k} h_{k}}{h_{i}}\right)\left(\sum_{k \neq j} \frac{a_{j k} x_{k}}{x_{j}} \frac{b_{i k} y_{k}}{y_{j}} \cdots \frac{k_{j k} h_{k}}{h_{j}}\right)\right]^{\frac{1}{2}}\right\} \\
\leq \max _{i \neq j} \frac{1}{2}\left\{a_{i i} b_{i i} \cdots k_{i i}+a_{j j} b_{j j} \cdots k_{j j}+\left[\left(a_{i i} b_{i i} \cdots k_{i i}-a_{j j} b_{j j} \cdots k_{j j}\right)^{2}\right.\right. \\
\left.\left.\quad+4\left(\sum_{k \neq i} \frac{a_{i k} x_{k}}{x_{i}} \sum_{k \neq i} \frac{b_{i k} y_{k}}{y_{i}} \cdots \sum_{k \neq i} \frac{k_{i k} h_{k}}{h_{i}}\right)\left(\sum_{k \neq j} \frac{a_{j k} x_{k}}{x_{j}} \sum_{k \neq j} \frac{b_{i k} y_{k}}{y_{j}} \cdots \sum_{k \neq j} \frac{k_{j k} h_{k}}{h_{j}}\right)\right]^{\frac{1}{2}}\right\} \\
=\max _{i \neq j} \frac{1}{2}\left\{a_{i i} b_{i i} \cdots k_{i i}+a_{j j} b_{j j} \cdots k_{j j}+\left[\left(a_{i i} b_{i i} \cdots k_{i i}-a_{j j} b_{j j} \cdots k_{j j}\right)^{2}\right.\right. \\
\left.\left.\quad+4\left(d_{i i} s_{i i} \cdots t_{i i}\right)\left(d_{j j} s_{j j} \cdots t_{j j}\right)\left(\rho^{2}\left(J_{A_{1}}\right) \rho^{2}\left(J_{A_{2}}\right) \cdots \rho^{2}\left(J_{A_{k}}\right)\right)\right]^{\frac{1}{2}}\right\} .
\end{aligned}
$$


If $A_{1} \circ A_{2} \circ \cdots \circ A_{k}$ is reducible, then substituting $A_{1}+t P, A_{2}+t P, \ldots, A_{k}+t P$ for $A_{1}, A_{2}, \ldots, A_{k}$, respectively, in the previous case, letting $t \rightarrow 0$, the result is derived.

Setting $k=2$ in Theorem 2.4, we have the following corollary.

Corollary 2.4 ([1]) Let $A_{1}, A_{2} \in M_{n}$ and $A_{1} \geq 0, A_{2} \geq 0$. Then

$$
\rho\left(A_{1} \circ A_{2}\right) \leq \max _{i \neq j} \frac{1}{2}\left\{a_{i i} b_{i i}+a_{j j} b_{j j}+\left[\left(a_{i i} b_{i i}-a_{j j} b_{j j}\right)^{2}+4 d_{i i} s_{i i} d_{j j} s_{j j} \rho^{2}\left(J_{A_{1}}\right) \rho^{2}\left(J_{A_{2}}\right)\right]^{\frac{1}{2}}\right\} .
$$

We next give a comparison between the upper bound in (2.4) and the upper bound in (2.5). Without loss of generality, for $i \neq j$, assume that

$$
\begin{aligned}
& a_{i i} b_{i i} \cdots k_{i i}+d_{i i} s_{i i} \cdots t_{i i} \rho\left(J_{A_{1}}\right) \rho\left(J_{A_{2}}\right) \cdots \rho\left(J_{A_{k}}\right) \\
& \quad \geq a_{j j} b_{j j} \cdots k_{j j}+d_{j j} s_{j j} \cdots t_{j j} \rho\left(J_{A_{1}}\right) \rho\left(J_{A_{2}}\right) \cdots \rho\left(J_{A_{k}}\right) .
\end{aligned}
$$

Let $\gamma=a_{i i} b_{i i} \cdots k_{i i}+a_{j j} b_{j j} \cdots k_{j j}$. From (2.5), we have

$$
\begin{aligned}
a_{i i} b_{i i} \cdots k_{i i}+a_{j j} b_{j j} \cdots k_{j j}+\left[\left(a_{i i} b_{i i} \cdots k_{i i}-a_{j j} b_{j j} \cdots k_{j j}\right)^{2}\right. \\
\left.+4\left(d_{i i} s_{i i} \cdots t_{i i}\right)\left(d_{j j} s_{j j} \cdots t_{j j}\right)\left(\rho^{2}\left(J_{A_{1}}\right) \rho^{2}\left(J_{A_{2}}\right) \cdots \rho^{2}\left(J_{A_{k}}\right)\right)\right]^{\frac{1}{2}} \\
\leq \gamma+\left\{\left(a_{i i} b_{i i} \cdots k_{i i}-a_{j j} b_{j j} \cdots k_{j j}\right)^{2}+4 d_{i i} s_{i i} \cdots t_{i i} \rho\left(J_{A_{1}}\right) \rho\left(J_{A_{2}}\right) \cdots \rho\left(J_{A_{k}}\right)\right. \\
\left.\quad \times\left[d_{i i} s_{i i} \cdots t_{i i} \rho\left(J_{A_{1}}\right) \rho\left(J_{A_{2}}\right) \cdots \rho\left(J_{A_{k}}\right)+a_{i i} b_{i i} \cdots k_{i i}-a_{j j} b_{j j} \cdots k_{j j}\right]\right\}^{\frac{1}{2}} \\
=\gamma+\left[\left(a_{i i} b_{i i} \cdots k_{i i}-a_{j j} b_{j j} \cdots k_{j j}+2 d_{i i} s_{i i} \cdots t_{i i} \rho\left(J_{A_{1}}\right) \rho\left(J_{A_{2}}\right) \cdots \rho\left(J_{A_{k}}\right)\right)^{2}\right]^{\frac{1}{2}} \\
=2 a_{i i} b_{i i} \cdots k_{i i}+2 d_{i i} s_{i i} \cdots t_{i i} \rho\left(J_{A_{1}}\right) \rho\left(J_{A_{2}}\right) \cdots \rho\left(J_{A_{k}}\right) .
\end{aligned}
$$

Thus, from (2.5) and the above inequality, we have

$$
\begin{aligned}
\rho\left(A_{1} \circ A_{2} \circ \cdots \circ A_{k}\right) \\
\leq \max _{i \neq j} \frac{1}{2}\left\{a_{i i} b_{i i} \cdots k_{i i}+a_{j j} b_{j j} \cdots k_{j j}+\left[\left(a_{i i} b_{i i} \cdots k_{i i}-a_{j j} b_{j j} \cdots k_{j j}\right)^{2}\right.\right. \\
\left.\left.\quad+4\left(d_{i i} s_{i i} \cdots t_{i i}\right)\left(d_{j j} s_{j j} \cdots t_{j j}\right)\left(\rho^{2}\left(J_{A_{1}}\right) \rho^{2}\left(J_{A_{2}}\right) \cdots \rho^{2}\left(J_{A_{k}}\right)\right)\right]^{\frac{1}{2}}\right\} \\
\leq \max _{1 \leq i \leq n} \frac{1}{2}\left(2 a_{i i} b_{i i} \cdots k_{i i}+2 d_{i i} s_{i i} \cdots t_{i i} \rho\left(J_{A_{1}}\right) \rho\left(J_{A_{2}}\right) \cdots \rho\left(J_{A_{k}}\right)\right) \\
=\max _{1 \leq i \leq n}\left(a_{i i} b_{i i} \cdots k_{i i}+d_{i i} s_{i i} \cdots t_{i i} \rho\left(J_{A_{1}}\right) \rho\left(J_{A_{2}}\right) \cdots \rho\left(J_{A_{k}}\right)\right) .
\end{aligned}
$$

Hence, the upper bound (2.5) is better than bound (2.4). Here too, we could not give the comparison between (2.4) and (2.3) or (2.5) and (2.3). Next, we give an example which shows that the results obtained in Theorems 2.3 and 2.4 are better than inequalities (2.3).

Let

$$
A=\left(\begin{array}{cccc}
2 & 0 & 1 & 1 \\
1 & 4 & 0.5 & 0.5 \\
1 & 0 & 3 & 0.5 \\
0.5 & 1 & 1 & 2
\end{array}\right), \quad B=\left(\begin{array}{cccc}
2 & 0.5 & 0.5 & 0.5 \\
1 & 1 & 1 & 1 \\
0.5 & 0 & 2 & 0.5 \\
0 & 1 & 1 & 2
\end{array}\right)
$$




$$
C=\left(\begin{array}{llll}
4 & 1 & 1 & 1 \\
2 & 2 & 1 & 1 \\
0 & 2 & 2 & 1 \\
1 & 1 & 1 & 1
\end{array}\right), \quad D=\left(\begin{array}{cccc}
1 & 0.5 & 2 & 0.5 \\
0.5 & 1 & 0.5 & 0 \\
0 & 0.5 & 1 & 0.5 \\
0 & 1 & 0.5 & 1
\end{array}\right)
$$

Let $A \circ B \circ C \circ D=P=\left(p_{i j}\right)$. Then

$$
P=\left(\begin{array}{cccc}
16 & 0 & 1 & 0.25 \\
1 & 8 & 0.25 & 0 \\
0 & 0 & 12 & 0.075 \\
0 & 1 & 0.5 & 4
\end{array}\right), \quad A B C D=\left(\begin{array}{cccc}
35.5 & 55.75 & 86 & 35.75 \\
57.75 & 91.875 & 139 & 57.875 \\
30.25 & 57.25 & 78 & 34.75 \\
34.875 & 64.125 & 87.5 & 38.875
\end{array}\right) \text {. }
$$

It is easy to calculate that $\rho(P)=16.0028$. By inequalities (2.4) and (2.5), we have

$$
\rho(P) \leq \max _{1 \leq i \leq 4}\left\{p_{i i}\left(1+\rho\left(J_{A}\right) \rho\left(J_{B}\right) \rho\left(J_{C}\right) \rho\left(J_{D}\right)\right)\right\}=36.2262
$$

and

$$
\begin{aligned}
\rho(P) & \leq \max _{i \neq j} \frac{1}{2}\left\{p_{i i}+p_{i j}+\left[\left(p_{i i}-p_{j j}\right)^{2}+4 p_{i i} p_{j i} \rho^{2}\left(J_{A}\right) \rho^{2}\left(J_{B}\right) \rho^{2}\left(J_{C}\right) \rho^{2}\left(J_{D}\right)\right]^{\frac{1}{2}}\right\} \\
& =29.6605 .
\end{aligned}
$$

By inequality (2.3) and Lemma 2.1, we have $\rho(P) \leq 91.875$.

\section{Conclusions}

In this paper, we have proposed some upper bounds for $\rho\left(A_{1} \circ A_{2} \circ \cdots \circ A_{k}\right)$ of the Hadamard product of matrices. These bounds generalize some corresponding results of [1-4].

\section{Acknowledgements}

This research is financed by the Natural Science Foundation of Shandong Province ZR2017MA050; Natural Science Foundation of Zhejiang Province (LY14A010007) and Ningbo Natural Science Foundation (2015A610173).

\section{Competing interests}

The authors declare that they have no competing interests.

Authors' contributions

All authors contributed equally to the writing of this paper. All authors read and approved the final manuscript.

\section{Author details}

${ }^{1}$ College of Mathematics Science, Dezhou University, Dezhou, Shandong 253023, People's Republic of China.

²Department of Mathematics, Zhejiang Wanli University, Ningbo, Zhejiang 315100, People's Republic of China.

\section{Publisher's Note}

Springer Nature remains neutral with regard to jurisdictional claims in published maps and institutional affiliations.

Received: 1 October 2017 Accepted: 19 December 2017 Published online: 05 January 2018

\section{References}

1. Fang, MZ: Bounds on eigenvalues of the Hadamard product and the Fan product of matrices. Linear Algebra Appl. 425, 7-15 (2007)

2. Liu, QB, Chen, GL: On two inequalities for the Hadamard product and the Fan product of matrices. Linear Algebra Appl. 431, 974-984 (2009)

3. Liu, QB, Chen, GL, Zhao, LL: Some new bounds on the spectral radius of matrices. Linear Algebra Appl. 432, 936-948 (2010) 
4. Huang, R: Some inequalities for the Hadamard product and the Fan product of matrices. Linear Algebra Appl. 428, 1551-1559 (2008)

5. Zhao, LL: Two inequalities for the Hadamard product of matrices. J. Inequal. Appl. 2012, 122 (2012)

6. Audenaert, KMR: Spectral radius of Hadamard product versus conventional product for non-negative matrices. Linear Algebra Appl. 432, 366-368 (2010)

7. Horn, RA, Zhang, FZ: Bounds on the spectral radius of a Hadamard product of nonnegative or positive semidefinite matrices. Electron. J. Linear Algebra 20, 90-94 (2010)

8. Huang, ZJ: On the spectral radius and the spectral norm of Hadamard products of nonnegative matrices. Linear Algebra Appl. 434, 457-462 (2011)

9. Zhou, DM, Chen, GL, Wu, GX, Zhang, XY: Some new bounds for eigenvalues of the Hadamard product and the Fan product of matrices. Linear Algebra Appl. 438, 1415-1426 (2013)

10. Zhou, DM, Chen, GL, Wu, GX, Zhang, XY: Some inequalities for the Hadamard product of an M-matrix and an inverse M-matrix. J. Inequal. Appl. 2013, 16 (2013)

11. Berman, A, Plemmons, RJ: Nonnegative Matrices in the Mathematical Sciences. SIAM, Philadelphia (1994)

12. Brauer, A: Limits for the characteristic roots of a matrix. Duke Math. J. 14, 21-26 (1947)

\section{Submit your manuscript to a SpringerOpen ${ }^{\circ}$} journal and benefit from:

- Convenient online submission

- Rigorous peer review

Open access: articles freely available online

High visibility within the field

- Retaining the copyright to your article

Submit your next manuscript at $\gg$ springeropen.com 